\title{
Superlattices
}

and Microstructures

ELSEVIER

Superlattices and Microstructures 36 (2004) 465-471

www.elsevier.com/locate/superlattices

\section{Theoretical assessment of electronic transport in $\mathrm{InN}$}

\author{
C. Bulutay ${ }^{\mathrm{a}, *}$, B.K. Ridley ${ }^{\mathrm{b}}$ \\ ${ }^{a}$ Department of Physics, Bilkent University, Ankara 06800, Turkey \\ ${ }^{\mathrm{b}}$ Department of Electronic Systems Engineering, University of Essex, Colchester C04 3SQ, UK
}

Available online 28 October 2004

\begin{abstract}
Among the group-III nitrides, InN displays markedly unusual electronic transport characteristics due to its smaller effective mass, high peak velocity and high background electron concentration. First, a non-local empirical pseudopotential band structure of $\mathrm{InN}$ is obtained in the light of recent experimental and first-principles results. This is utilized within an ensemble Monte Carlo framework to illuminate the interesting transport properties. It is observed that $\mathrm{InN}$ has a peak velocity which is about $75 \%$ higher than that of $\mathrm{GaN}$ while at higher fields its saturation velocity is lower than that of $\mathrm{GaN}$. Because of the strongly degenerate regime brought about by the high background electron concentration, the electron-electron interaction is also investigated, but its effect on the steady-state and transient velocity-field characteristics is shown to be negligible. Finally, hot phonon generation due to excessive polar optical phonon production in the electron scattering and relaxation processes is accounted for. The main findings are the appreciable reduction in the saturation drift velocity and the slower recovery from the velocity overshoot regime. The time evolution of the hot phonon distribution is analysed in detail and it is observed to be extremely anisotropic, predominantly along the electric force direction.
\end{abstract}

(C) 2004 Elsevier Ltd. All rights reserved.

\section{Introduction}

InN offers interesting electronic transport characteristics due to its smaller effective mass and high peak velocity, in addition to its bright optoelectronic prospects. While the

\footnotetext{
* Corresponding author.

E-mail address: bulutay@fen.bilkent.edu.tr (C. Bulutay).
} 
recent achievements in uncovering the structural and optical properties of $\mathrm{InN}$ have been significant, the areas of carrier dynamics and relaxation characteristics are still immature. However, these properties are bound to become important, even in photonics applications, such as in the promising $\mathrm{Ga}_{1-x} \mathrm{In}_{x} \mathrm{~N}$ heterostructure lasers.

In this work, we assess the electronic transport properties of InN using the ensemble Monte Carlo (EMC) technique. There have been a number of similar treatments in the past [1,2]. However, these results are now challenged in the light of new band structure data for the recently obtained high-purity InN films [3,4]. Furthermore, even in these not intentionally doped samples the background electron concentration is quite high [5]. Such high densities are also inevitable in heterostructure lasers and high-electron-mobility transistors. Therefore, their effects on carrier transport deserve to be thoroughly studied.

A direct implication of the high electronic densities is the enhancement of the electron-electron interaction. Its seemingly indirect but actually more important consequence is the proliferation of optical phonons in such polar semiconductors during the carrier relaxation process under high fields. These so-called hot phonons in turn have more drastic effects on carrier transport which we also investigate in this paper.

\section{Theoretical framework}

The predictive power of an EMC simulation directly depends on the representation of the underlying electronic band structure as well as the scattering mechanisms. As regards the former, the band structure for $\mathrm{InN}$ has been elusive, with ongoing debate on its fundamental bandgap. After accumulating convincing evidence for a bandgap value of around $0.7-0.8 \mathrm{eV}[3,4]$, we obtained a non-local empirical pseudopotential (NLEP) band structure for InN fitted to very recently obtained first-principles results [6], which is shown in Fig. 1. An important quantity for the high-field transport is the density of states (DOS), which plays a decisive role in a number of scattering mechanisms. While using the DOS based on the NLEP method for producing the scattering tables, we still need its faithful valley-based representation in order to have an efficient simulation tool. This is illustrated in Fig. 2, which reveals a close agreement between the two up to $4 \mathrm{eV}$ above the conduction band minimum.

With the current technology, as-grown $\mathrm{InN}$ is always n-type with a very high background carrier concentration (above $10^{18} \mathrm{~cm}^{-3}$ ) [5]. Therefore, the strongly degenerate regime where non-parabolicity, electron-electron interaction and hot phonon effects are essential needs to be considered. The band non-parabolocities are accurately taken into account as indicated by the close agreement with the actual DOS (cf. Fig. 2). Previously, the electron-electron interaction has been incorporated into the EMC framework at several levels of complexity [7]. Here, we favour the molecular dynamics technique [8,9] at the expense of its substantial computational cost, as it is free from the drawbacks of other traditional approaches which artificially separate the carrier-carrier interaction into short-range binary collisions and long-range plasmon scattering [9].

For hot phonon effects, we essentially follow along the lines of the pioneering work in this field [10]. However, instead of applying an isotropic hot phonon treatment which is justified under intense optical illumination, we have to consider the anisotropy in phonon 


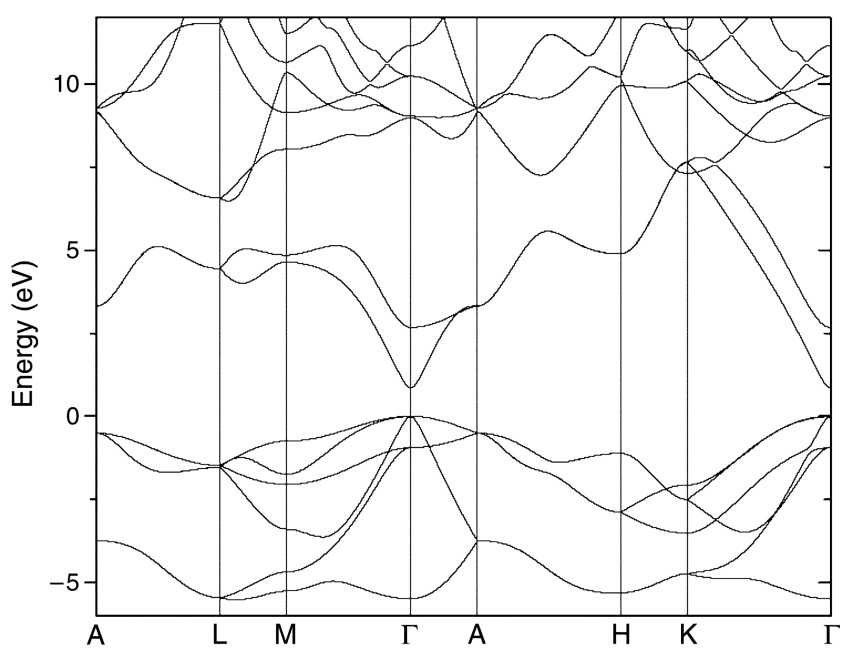

Fig. 1. The non-local empirical pseudopotential band structure for wurtzite InN.

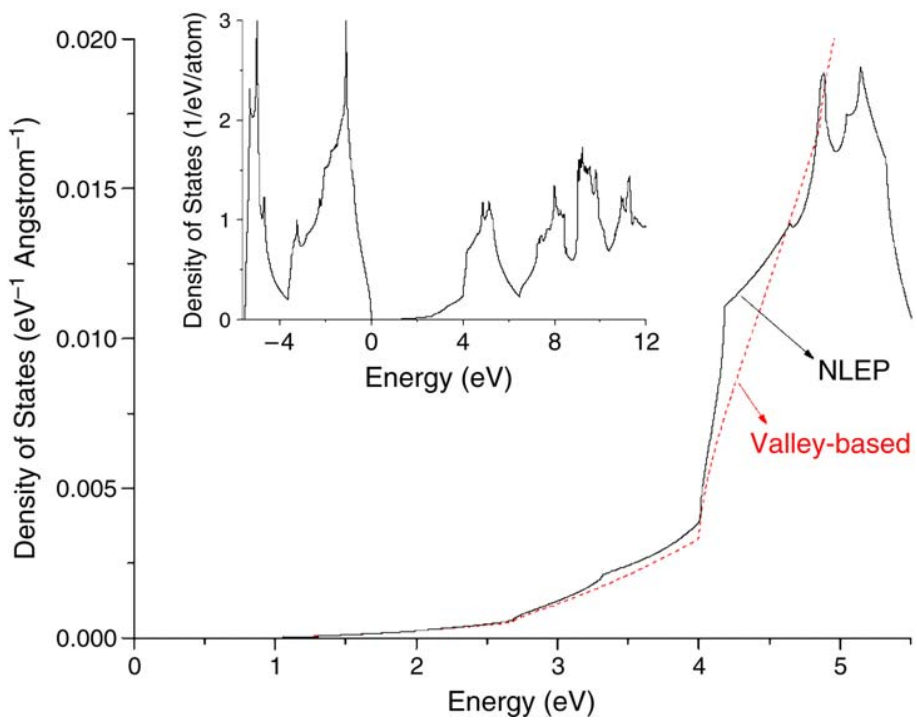

Fig. 2. The density of states for the band structure shown in Fig. 1 (solid) and its valley-based representation (dashed) in the vicinity of the conduction band edge. The inset includes the valence bands as well. The energy reference value is chosen as the valence band maximum.

production as dictated by the direction of the applied high electric field. On the more technical side, for the hot phonon population, we only consider the polar longitudinal optical (LO) branch, as it gives rise to the strongest electron scattering. An important 


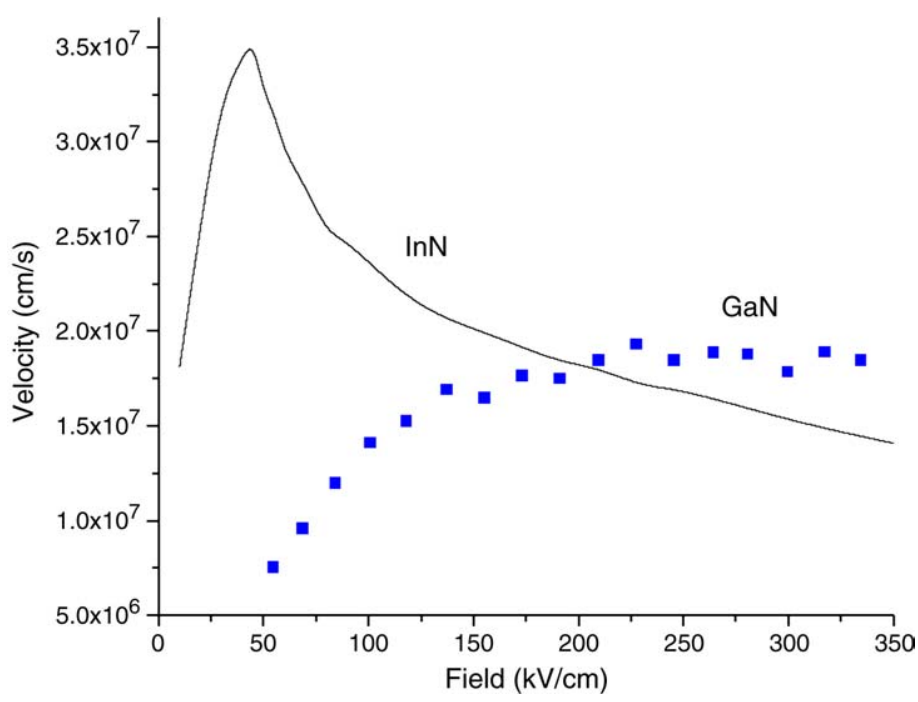

Fig. 3. The steady-state velocity-field characteristics of InN computed with the EMC method (solid). For comparison the measured values for GaN [12] are also shown (squares). Note that the InN results here do not include the hot phonon or electron-electron interaction effects (see the text) which can make a fair comparison difficult.

parameter here is the lifetime of this branch, which characterizes how fast these phonons decay into other phonons and reach an equilibrium distribution once the excitation is removed. As a standard assumption, we assume that these secondary phonons have even shorter lifetimes and moreover do not couple back to electrons effectively. This, then, allows us to use a constant LO phonon lifetime. Because of the lack of an experimental report of this quantity, we use the value of $\tau_{\mathrm{LO}}=3 \mathrm{ps}$, which applies to $\mathrm{GaN}$ at room temperature. Finally, working at high densities, the associated degeneracy effects also need to be accounted for, for instance using the Lugli-Ferry recipe [11].

\section{Results}

First, we would like to mention that our results to follow are obtained for a lattice temperature of $300 \mathrm{~K}$. In Fig. 3 we show the steady-state velocity-field characteristics of InN. The most important feature is the very high peak velocity of $\mathrm{InN}$, of about $3.5 \times 10^{7} \mathrm{~cm} / \mathrm{s}$ at a field of about $45 \mathrm{kV} / \mathrm{cm}$, beyond which the negative differential mobility region sets in. This peak field value is smaller than the previous EMC result $[1,2]$ of $65 \mathrm{kV} / \mathrm{cm}$, which we attribute to the poor band structure and effective mass parameters that were established at the time of those publications $[1,2]$. Compared with the measured values for $\mathrm{GaN}$ [12], the peak velocity of $\mathrm{InN}$ is about $75 \%$ higher, whereas the saturation velocity is lower than that of GaN. In these steady-state characteristics, the effects of hot phonons and the electron-electron interaction are not included. Their roles are displayed in Fig. 4, which shows the transient response characteristics of $\mathrm{InN}$ at an applied 


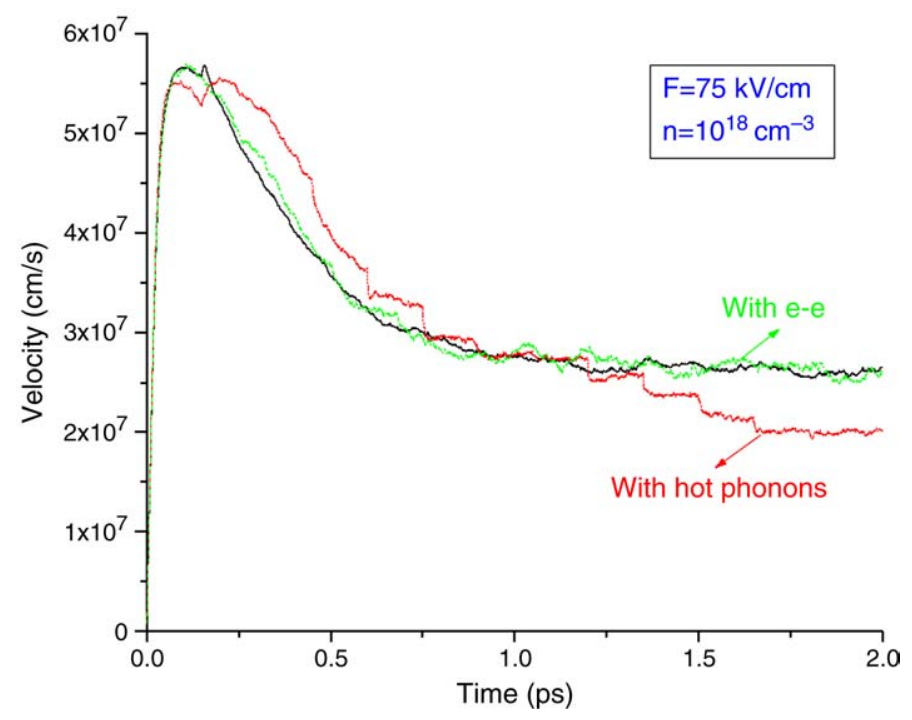

Fig. 4. Transient velocity response characteristics of InN computed using the EMC method under an applied field of $75 \mathrm{kV} / \mathrm{cm}$ and for an electron concentration of $10^{18} \mathrm{~cm}^{-3}$. Three simulations are compared: with hot phonon effects included; with electron-electron interaction included; and without these two effects.

field of $75 \mathrm{kV} / \mathrm{cm}$, and an electron density of $10^{18} \mathrm{~cm}^{-3}$. It is observed that a strong velocity overshoot takes place at about $0.2 \mathrm{ps}$ after the application of the field. The electron-electron interaction has no discernible effect on the transient characteristics, as expected. On the other hand, the presence of hot phonons leads to slightly slower recovery from the overshoot regime and, more importantly, causes an appreciable reduction in the saturation velocity compared to its value in Fig. 3. This final observation needs to be taken into account in the development of prospective InN-based devices such as field-effect transistors and heterostructure lasers.

Finally, choosing an electric field value of $75 \mathrm{kV} / \mathrm{cm}$ which lies in the negative differential mobility region, we investigate the time evolution of the hot phonon radial and polar distributions. In Fig. 5, the hot phonon distribution as a function of the magnitude of the phonon wavevector is shown for three different time instants: $0.2,0.5$ and 1 ps. For comparison, the equilibrium phonon distribution is also included. It can be observed that such a high field quickly drives the phonon population out of equilibrium and these are mainly the long-wavelength phonons due to the well-known nature of the Fröhlich interaction. For all these curves, the phonon wavevector is chosen to be along the electric force direction. As a matter of fact, the phonon distribution is quite anisotropic. This is illustrated in Fig. 6 which displays the hot phonon distribution versus the polar angle measured with respect to the electric force direction. The hot phonon distribution is remarkably inflated along the electric force direction due to forward-scattering behaviour of the polar optical phonon emission. This precludes a simplistic isotropic treatment of hot phonons under high-field transport conditions. 


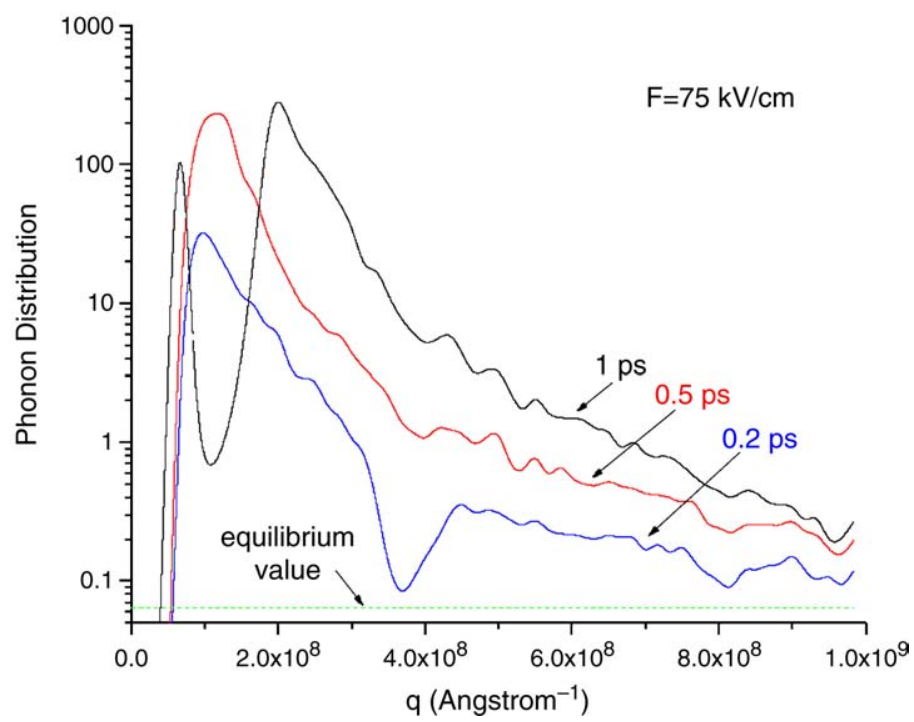

Fig. 5. The hot phonon distribution (on a logarithmic scale) versus the magnitude of the phonon wavevector, computed at an electric field of $75 \mathrm{kV} / \mathrm{cm}$; B-spline fittings to suppress the noise are shown. The phonon wavevector is chosen to be along the force direction. The time evolution is demonstrated at three different time instants as labelled, together with the equilibrium phonon distribution (dashed).

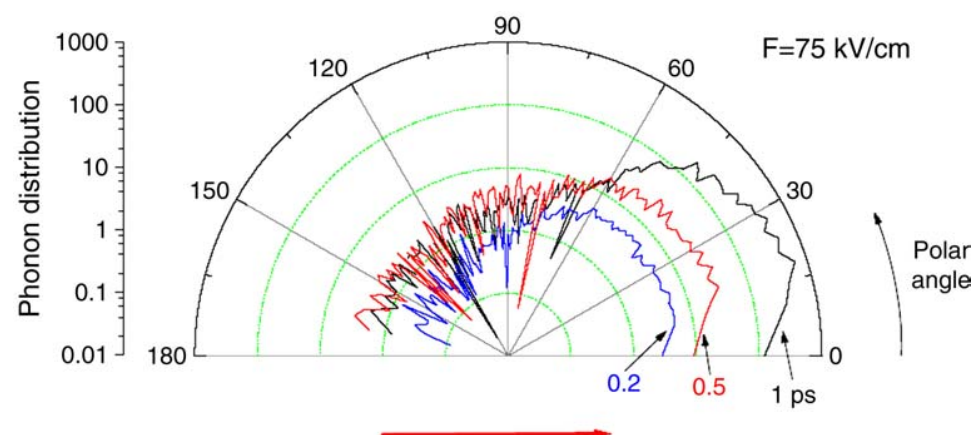

Electric Force

Fig. 6. The hot phonon distribution versus the polar angle (in degrees) measured with respect to the electric force direction at an electric field of $75 \mathrm{kV} / \mathrm{cm}$ (note the logarithmic scale). Time evolution is shown at three different time instants, as labelled.

\section{Conclusion}

In this work, the steady-state velocity-field characteristics of InN are obtained using the ensemble Monte Carlo technique based on more reliable band structure data. Notably, InN possesses a peak drift velocity that is $75 \%$ higher than that of $\mathrm{GaN}$ but has a lower saturation velocity at higher fields. An important consequence of the very high electronic 
concentration is noted to be the hot phonon production which reduces the saturation drift velocity and leads to slower recovery from the velocity overshoot regime. The time evolution of the hot phonon distribution is analysed in detail and is observed to be extremely anisotropic, predominantly along the electric force direction.

\section{Acknowledgment}

C.B. would like to acknowledge the support of The Scientific and Technical Research Council of Turkey (TÜBİTAK) within the COST 288 Action.

\section{References}

[1] E. Bellotti, B.K. Doshi, K.F. Brennan, J.D. Albrecht, P.P. Ruden, J. Appl. Phys. 85 (1999) 916.

[2] B.E. Foutz, S.K. O’Leary, M.S. Shur, L.F. Eastman, J. Appl. Phys. 85 (1999) 7727.

[3] V.Yu. Davydov, A.A. Klochikhin, R.P. Seisyan, V.V. Emtsev, S.V. Ivanov, F. Bechstedt, J. Furthmüller, H. Harima, A.V. Mudryi, J. Aderhold, O. Semchinova, J. Graul, Phys. Status Solidi b 229 (2002) R1.

[4] J. Wu, W. Walukiewicz, K.M. Yu, J.W. Ager III, E.E. Haller, H. Lu, W.J. Schaff, Y. Saito, Y. Nanishi, Appl. Phys. Lett. 80 (2002) 3967.

[5] A.G. Bhuiyan, A. Hashimoto, A. Yamamoto, J. Appl. Phys. 94 (2003) 2779.

[6] S.-H. Wei, P. Carrier, 2004, cond-mat/0401306.

[7] D.K. Ferry, Semiconductor Transport, Taylor \& Francis, London, 2000.

[8] W.J. Gross, D. Vasileska, D.K. Ferry, IEEE Electron Device Lett. 20 (1999) 463.

[9] D. Vasileska, S.M. Goodnick, Mater. Sci. Eng. R 38 (2002) 181.

[10] P. Lugli, C. Jacoboni, L. Reggiani, P. Kocevar, Appl. Phys. Lett. 50 (1987) 1251.

[11] P. Lugli, D.K. Ferry, IEEE Trans. Electron Devices 32 (1985) 2431.

[12] M. Wraback, H. Shen, J.C. Carrano, T. Li, J.C. Campbell, M.J. Schurman, I.T. Ferguson, Appl. Phys. Lett. 76 (2000) 1155. 